\title{
Magnetocaloric effect in one-dimensional antiferromagnets
}

\author{
M. E. Zhitomirsky \\ Commissariat à l'Energie Atomique, DSM/DRFMC/SPSMS, 17 avenue des Martyrs, \\ 38054 Grenoble, Cédex 9 France
}

\begin{abstract}
A. Honecker
Technische Universität Braunschweig, Institut für Theoretische Physik, Mendelsohnstrasse 3, 38106 Braunschweig, Germany
\end{abstract}

30 April 2004; revised 8 July 2004

\begin{abstract}
An external magnetic field induces large relative changes in the entropy of one-dimensional quantum spin systems at finite temperatures. This leads to a magnetocaloric effect, i.e. a change in temperature during an adiabatic (de)magnetization process. Several examples of one-dimensional spin- $1 / 2$ models are studied by employing the Jordan-Wigner transformation and exact diagonalization. During an adiabatic (de)magnetization process temperature drops in the vicinity of a fieldinduced zero-temperature quantum phase transition. Comparing different levels of frustration, we find that more frustrated systems cool down to lower temperatures. For geometrically frustrated spin models a finite entropy survives down to zero temperature at certain magnetic fields. This property suggests frustrated quantum spin systems as promising alternative refrigerant materials for low-temperature magnetic refrigeration.

PACS numbers: 75.30.Sg, 75.10.Jm, 75.50.Ee
\end{abstract}

\section{Introduction}

The magnetocaloric effect, or heating/cooling of a magnetic system under an adiabatic (de)magnetization process, is related to the isothermal variation of the entropy in a magnetic field via a simple thermodynamic relation

$$
\left(\frac{\partial T}{\partial H}\right)_{S}=-T \frac{(\partial S / \partial H)_{T}}{C},
$$

where $C$ is the specific heat at a constant magnetic field. A field variation of entropy can be found via a temperature variation of the magnetization: $(\partial S / \partial H)_{T}=(\partial M / \partial T)_{H}$. Standard examples of magnetic materials with a significant magnetocaloric effect include paramagnetic salts, which consist of noninteracting spins, and ferromagnets near the Curie point, where $(\partial M / \partial T)_{H}$ is, generally, large [1,2]. Besides a fundamental interest, the magnetocaloric effect has a great importance for the technique of magnetic cooling, 
which has been employed over the years to reach temperatures in a sub-Kelvin range [3]. A certain progress has also been achieved to utilize this technique for room temperature refrigeration [4, 5].

Recently, it has been predicted that an enhanced magnetocaloric effect exists in the vicinity of finite-field transitions in a class of geometrically frustrated antiferromagnets 6]. Local spins in such magnets reside on special types of crystal lattices, with pyrochlore, kagome, and garnet lattices being a few well-known examples. Competing antiferromagnetic interactions on frustrated lattices lead to a macroscopic degeneracy (entropy) of classical ground states. Large variations of the total entropy occur near the saturation field $H_{c}$, where the transition to a fully polarized nondegenerate state takes place. Above $H_{c}$ in the saturated phase, highly frustrated magnets have a flat dispersionless low-energy branch of magnons with energy $\left(H-H_{c}\right)$. Dispersionless excitations can be represented in real space as localized quasiparticles. Such localized magnon excitations have recently also been studied in frustrated quantum spin models [7.8] where it was shown that the corresponding many-particle states are not only exact eigenstates of the Hamiltonian, but indeed the lowest ones. Hence, the macroscopic zero$T$ entropy of geometrically frustrated magnets at $H=H_{c}$ is robust against quantum fluctuations [9] (see also [10]), whereas below $H_{c}$ the quantum (or thermal) order by disorder effect should lift the classical degeneracy [7,11]. It is the condensation of a macroscopic number of localized magnons near the saturation field, which leads to the enhanced magnetocaloric effect in geometrically frustrated magnets [6].

The aim of the present work is two-fold. First, we argue that a large magnetocaloric effect appears near continuous phase transitions in a magnetic field for general onedimensional (1D) quantum spin systems. This phenomenon has a simple physical origin. The excitation spectrum at the quantum critical point is different and, generally, softer if compared to spectra above and below the transition. The density of low-energy modes is strongly enhanced in spin chains due to the 1D van Hove singularity. Therefore, the soft modes increase the total magnetic entropy around the transition point and produce a sizeable magnetocaloric effect. We present analytic calculations for two exactly solved models: an $X Y$ antiferromagnetic chain with a magnetic field applied perpendicular to the plane and an Ising model in a transverse field. Then, we study by means of exact diagonalization of finite lattices a spin-1/2 Heisenberg chain. Secondly, we investigate how the magnetocaloric effect in 1D systems changes with increasing degree of frustration. For this purpose we consider a frustrated $J_{1}-J_{2}$ spin- $1 / 2$ chain and the so-called sawtooth spin chain as a 1D toy model for the class of geometrically frustrated lattices containing the kagome and pyrochlore lattice. 


\section{2. $X Y$ chain in transverse field}

We begin with a simple spin-1/2 XY antiferromagnet in a transverse magnetic field, which is described by the Hamiltonian:

$$
\hat{\mathcal{H}}=\sum_{i=1}^{N}\left[J\left(S_{i}^{x} S_{i+1}^{x}+S_{i}^{y} S_{i+1}^{y}\right)-H S_{i}^{z}\right] .
$$

With the help of the Jordan-Wigner transformation [12] this is mapped to a Hamiltonian of free spinless fermions:

$$
\hat{\mathcal{H}}=-\frac{H N}{2}+\sum_{k} \varepsilon_{k} c_{k}^{\dagger} c_{k}, \quad \varepsilon_{k}=H+J \cos k .
$$

In high magnetic fields the ground state is the fully polarized spin state, which describes

the fermion vacuum. As the magnetic field is lowered, the excitation gap for fermions decreases and vanishes at the saturation field $H_{c}=J$. At the transition point the low-energy fermions have a parabolic dispersion $\varepsilon_{\pi+q} \approx \frac{1}{2} J q^{2}$ for $|q| \ll 1$.

The heat capacity of a gas of free fermions is

$$
C=\frac{1}{T^{2}} \sum_{k} \varepsilon_{k}^{2} n_{k}\left(1-n_{k}\right)
$$

where $n_{k}=\left[\mathrm{e}^{\varepsilon_{k} / T}+1\right]^{-1}$ are the occupation numbers. At the saturation field the specific heat has a square-root low-temperature dependence

$$
C / N=\frac{3}{4} \zeta\left(\frac{3}{2}\right)\left(1-\frac{1}{\sqrt{2}}\right) \sqrt{\frac{T}{2 \pi J}}
$$

with $\zeta\left(\frac{3}{2}\right) \approx 2.612375$.

The magnetization of an $X Y$ chain is given by $M=\frac{1}{2} N-\sum_{k} n_{k}$, which reduces at zero temperature to

$$
M / N=\frac{1}{2}-\frac{1}{\pi} \arccos \left(H / H_{c}\right)
$$

(compare with eq. (3.5) of [13]). The magnetization curve $M(H)$ has a standard squareroot singularity near the saturation field [14,15]. The magnetocaloric effect is calculated as

$$
\left(\frac{\partial S}{\partial H}\right)_{T}=-\frac{1}{T^{2}} \sum_{k} \varepsilon_{k} n_{k}\left(1-n_{k}\right) .
$$

At $H=H_{c}$ and low temperatures one finds

$$
\begin{aligned}
& \left(\frac{\partial S}{\partial H}\right)_{T}=-\frac{c_{1} N}{2 \sqrt{2 \pi T J}}, \\
& c_{1}=\frac{2}{\sqrt{\pi}} \int_{0}^{\infty} \frac{d x}{\mathrm{e}^{x^{2}}+1}=\sum_{n=1}^{\infty} \frac{(-1)^{n-1}}{n^{1 / 2}} \approx 0.604899 .
\end{aligned}
$$




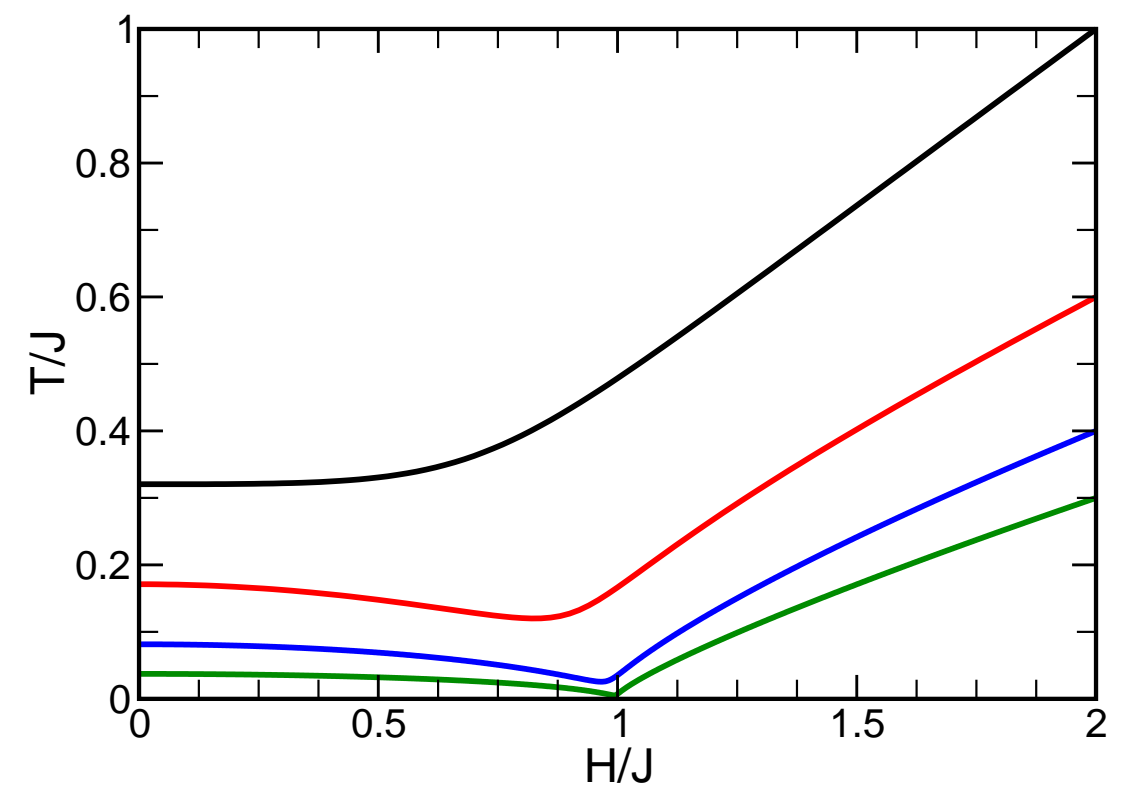

Figure 1. Adiabatic demagnetization curves of the $X Y$ antiferromagnetic $S=1 / 2$ chain.

The low-temperature expressions for the entropy above, at and below the saturation field $H_{c}$ are given by

$$
\begin{array}{rlrl}
S / N & =\frac{\Delta_{H}}{\sqrt{2 \pi T}} \mathrm{e}^{-\Delta_{H} / T}, & H>H_{c}, \\
S / N=\frac{3}{2} \zeta\left(\frac{3}{2}\right)\left(1-2^{-1 / 2}\right) \sqrt{\frac{T}{2 \pi J}}, & H=H_{c}, \\
S / N=\frac{\pi}{3} \frac{T}{\sqrt{2\left|\Delta_{H}\right| J}}, & H<H_{c},
\end{array}
$$

where $\Delta_{H}=H-H_{c}$ and $\left|\Delta_{H}\right| \gg T$ is assumed. At $T=0$ in an arbitrary magnetic field the system has a unique quantum ground state and, correspondingly, zero total entropy. At $T>0$, thermal fluctuations yield different entropy contributions in the above three regimes. Variation of the applied magnetic field leads, therefore, to large relative changes in the total entropy and to a large magnetocaloric effect.

The adiabatic demagnetization curves of an $X Y$ antiferromagnet can be found either from a direct numerical solution of $S(H, T)=$ const or by numerical integration of the differential relation (11). The results are presented in Fig. 1 for $H_{i}=2 H_{c}$ and several starting temperatures $T_{i}$. At $H=H_{c}$ the cooling rate $(\partial T / \partial H)_{S}$ has the maximal value determined by eqs. (11), (5) and (8) . The lowest temperature of an adiabatic process is reached at $H^{*}<H_{c}$. At low temperatures the difference between these two fields becomes, however, very small. Using eqs. (9) we find the following analytic expressions for a field-dependence of temperature. At $\left(H-H_{c}\right) \gg T$, temperature changes nearly 
linearly with $\left(H-H_{c}\right)$ due to a dominant exponential contribution. In this region

$$
\frac{1}{T}\left(\frac{\partial T}{\partial H}\right)_{S}=\frac{1}{H-H_{c}}
$$

The lowest temperature reachable during an adiabatic demagnetization process from $T_{i}$ and $H_{i}>H_{c}$ to $H_{f}=H_{c}$ is exponentially small:

$$
T_{f} \sim \frac{\left(H_{i}-H_{c}\right)^{2}}{T_{i}} \exp \left[-\frac{2\left(H_{i}-H_{c}\right)}{T_{i}}\right] .
$$

Below the saturation field, temperature decreases as $\left(H_{c}-H\right)^{1 / 2}$ with $H \rightarrow H_{c}$ $\left[\left(H_{c}-H\right) \gg T\right]$. In this region one finds

$$
\frac{1}{T}\left(\frac{\partial T}{\partial H}\right)_{S}=\frac{1}{2} \frac{1}{H-H_{c}} \text {. }
$$

The lowest temperature reachable during adiabatic magnetization from $H=H_{i}<H_{c}$ to $H_{f}=H_{c}$ is

$$
T_{f} \sim \frac{T_{i}^{2}}{H_{c}-H_{i}}
$$

\section{Heisenberg chain in magnetic field}

We now discuss the effect of $S^{z}-S^{z}$ interaction on the magnetothermodynamics of the phase transition in the spin- $1 / 2 X X Z$ chain:

$$
\hat{\mathcal{H}}=\sum_{i}\left[J\left(S_{i}^{x} S_{i+1}^{x}+S_{i}^{y} S_{i+1}^{y}+\Delta S_{i}^{z} S_{i+1}^{z}\right)-H S_{i}^{z}\right] .
$$

The case $\Delta=1$ corresponds to the Heisenberg chain. The interaction of the $z$ components shifts the fermion energies and the saturation field:

$$
\varepsilon_{k}=H-J \Delta+J \cos k, \quad H_{c}=J(1+\Delta) .
$$

It also induces an interaction between fermions:

$$
\begin{aligned}
V & =J \Delta \sum_{i} c_{i}^{\dagger} c_{i} c_{i+1}^{\dagger} c_{i+1} \\
& =\frac{1}{2 N} \sum_{k, k^{\prime}, q} J \Delta\left[\cos q-\cos \left(k-k^{\prime}+q\right)\right] c_{k+q}^{\dagger} c_{k^{\prime}-q}^{\dagger} c_{k^{\prime}} c_{k} .
\end{aligned}
$$

The interaction between spinless fermions vanishes as $k, k^{\prime}, q \rightarrow 0$. In the vicinity of the saturation field only wave-vectors near $k \approx \pi$ are important and, therefore, interactions play a minor role 14,15,16]. The main correction to the free energy at low temperatures is the Hartree-Fock contribution, which is estimated as

$$
\delta F_{\mathrm{HF}}=\langle V\rangle=J N \Delta\left(n^{2}-m^{2}\right)
$$

with $n=\left\langle c_{i}^{\dagger} c_{i}\right\rangle$ and $m=\left\langle c_{i}^{\dagger} c_{i+1}\right\rangle$. At the saturation field one finds

$$
\begin{aligned}
& n-m=\sum_{k} \frac{1-\cos k}{\mathrm{e}^{\varepsilon_{k} / T}+1}=2 c_{1} \sqrt{\frac{T}{2 \pi J}}, \\
& n+m=\sum_{k} \frac{1+\cos k}{\mathrm{e}^{\varepsilon_{k} / T}+1}=\frac{1}{2} \sqrt{\frac{T^{3}}{2 \pi J^{3}}} \zeta\left(\frac{3}{2}\right)\left(1-2^{-1 / 2}\right) .
\end{aligned}
$$


The temperature expansion of the free energy at $H=H_{c}$ is

$$
F / N=-\zeta\left(\frac{3}{2}\right)\left(1-2^{-1 / 2}\right)\left[\frac{T^{3 / 2}}{(2 \pi J)^{1 / 2}}-\Delta c_{1} \frac{T^{2}}{2 \pi J}\right],
$$

where the first $T^{3 / 2}$ term is a Fermi-gas contribution and the second term $T^{2}$ is the Hartree-Fock correction. The next order $T^{5 / 2}$ correction to eq. (17) is determined by deviations from a parabolic dispersion in $\varepsilon_{k}$. The interaction gives a negligible contribution in the thermodynamics of a Heisenberg chain at temperatures $T \lesssim 0.1 J$. The expression (17) agrees with the exact thermodynamic Bethe ansatz solution for a Heisenberg chain [12,17], which, however, gave the second $T^{2}$ term without numerical prefactor.

\section{Frustrated Heisenberg chain near saturation}

The density of low-energy states at the saturation field can be increased by frustration. As a first example we consider the $J_{1}-J_{2}$ chain with exchange interaction between the first and second neighbours at the point $J_{2}=\frac{1}{4} J_{1}$, where the dispersion degenerates from the generic quadratic behaviour to a fourth power, giving rise to a fourth-root instead of the usual square-root singularity in the $T=0$ magnetization curve at the saturation field [18, 19,20].

The $J_{1}-J_{2}$ Heisenberg chain is described by the Hamiltonian

$$
\hat{\mathcal{H}}=\sum_{i}\left[J_{1} \mathbf{S}_{i} \cdot \mathbf{S}_{i+1}+J_{2} \mathbf{S}_{i} \cdot \mathbf{S}_{i+2}-H S_{i}^{z}\right] .
$$

The fermion energies in the saturated phase are given by

$$
\varepsilon_{k}=H+J_{1}(\cos k-1)+J_{2}(\cos 2 k-1) .
$$

Frustrated interaction with the second neighbours does not change the saturation field value for $J_{2} \leq \frac{1}{4} J_{1}: H_{c}=2 J_{1}$. The long-wave length expansion of $\varepsilon_{k}$ near the minimum point yields

$$
\varepsilon_{\pi+q} \approx H-H_{c}+\left(\frac{J_{1}}{2}-2 J_{2}\right) q^{2}+\left(\frac{2 J_{2}}{3}-\frac{J_{1}}{24}\right) q^{4} .
$$

At the point $J_{2}=\frac{1}{4} J_{1}$, the first $q^{2}$-term vanishes and the dispersion of fermions is described by a quartic parabola: $\varepsilon_{\pi+q} \approx H-H_{c}+\frac{1}{8} J q^{4}\left(J_{1} \equiv J\right)$. This leads to a higher density of states above the gap $\rho(\omega) \sim \omega^{-3 / 4}$ than for the standard 1D van Hove singularity $\rho(\omega) \sim \omega^{-1 / 2}$. For a free fermion gas with a general dispersion law $\varepsilon_{k}=A k^{z}$, the entropy behaves as

$$
S / N=\frac{1+z}{\pi z} \Gamma\left(1+\frac{1}{z}\right) \zeta\left(1+\frac{1}{z}\right)\left(1-2^{-1 / z}\right) \frac{T^{1 / z}}{A^{1 / z}} .
$$

For $z=4$ this gives $S \sim T^{1 / 4}$. However, for $z>3$ the interaction between fermions becomes again a relevant perturbation at $H=H_{c}$ [16] such that eq. (20) is no longer expected to give the exact low-temperature asymptotics. Thermal fluctuations tend to reduce frustration by generating a temperature-dependent quadratic term 
$\varepsilon_{k} \approx B(T) k^{2}+A k^{z}$, which has to be determined self-consistently. In any case, we expect that at low temperatures the entropy of the frustrated chain is higher than the entropy of a Heisenberg chain. Since above $H_{c}$ the entropy has a similar exponential behaviour given by eq. (9), the frustrated chain has larger relative variations of the entropy and, consequently, should exhibit a larger magnetocaloric effect.

\section{Numerical results at the saturation field}

In this section we compare the analytic results for the $S=1 / 2$ Heisenberg chain to numerical results obtained by exact diagonalization of finite rings and check the scaling of the entropy for the $J_{1}-J_{2}$ Heisenberg model. A spectral representation is used for the evaluation of the thermodynamic quantities. In particular, the entropy is computed using

$$
S=\frac{1}{T Z} \sum_{n} E_{n} \mathrm{e}^{-E_{n} / T}+\ln Z,
$$

where $Z=\sum_{n} \mathrm{e}^{-E_{n} / T}$ is the partition function. The eigenvalues $E_{n}$ are obtained by full diagonalization of the Hamiltonian.

Since the CPU time for a full diagonalization grows as the third power of the dimension, it is important to use symmetries to reduce the dimension. We have used conservation of $S^{z}$, the $z$-component of the total spin, spin-inversion symmetry for $S^{z}=0$ and $S U(2)$-symmetry to reconstruct the spectra in the sector $S^{z}=1$. Then the biggest dimension arises for $S^{z}=2$. The actual size depends on the spatial symmetries, namely translations and reflections, which have also been exploited. Full diagonalizations have been performed with up to $N=20$ sites for all three spin- $1 / 2$ systems to be discussed below. The sawtooth chain has lowest spatial symmetry, hence the largest dimension occurs here, namely 12618 for $S^{z}=2$ and $N=20$. Since the magnetic field $H$ couples to the total magnetization $S^{z}$, which is a conserved quantity, one computation yields the dependence of the entropy $S$ both on temperature $T$ and the magnetic field $H$.

Fig. 2 presents a log-log plot of the temperature-dependence of the entropy of the Heisenberg chain and the $J_{1}-J_{2}$ chain with $J_{1}=J, J_{2}=\frac{1}{4} J$ at the saturation field $H_{c}=2 J$ for $N=12,16$, and 20. At low temperatures, the ground state at $H_{c}$ is twofold degenerate, i.e. $S=\ln 2$ for any $N$ as $T \rightarrow 0$. Accordingly, one observes finite-size effects in $S / N$ at low temperatures. At high temperatures, all curves recover the full entropy $S / N=\ln 2 \approx 0.6931$ of a spin- $1 / 2$ system. Finite-size effects are still negligible at intermediate temperatures and the numerical curves are straight lines in the log-log representation of Fig. 2. implying a power law for $S / N$ as a function of temperature. Next, we discuss these power laws in more detail and argue that the exponents are indeed the expected ones.

The bold solid line in Fig. 2 obtained from eq. (17) is (see also eqs. (91) and (201)

$$
S / N=0.457874(T / J)^{1 / 2}-0.1473254(T / J) .
$$




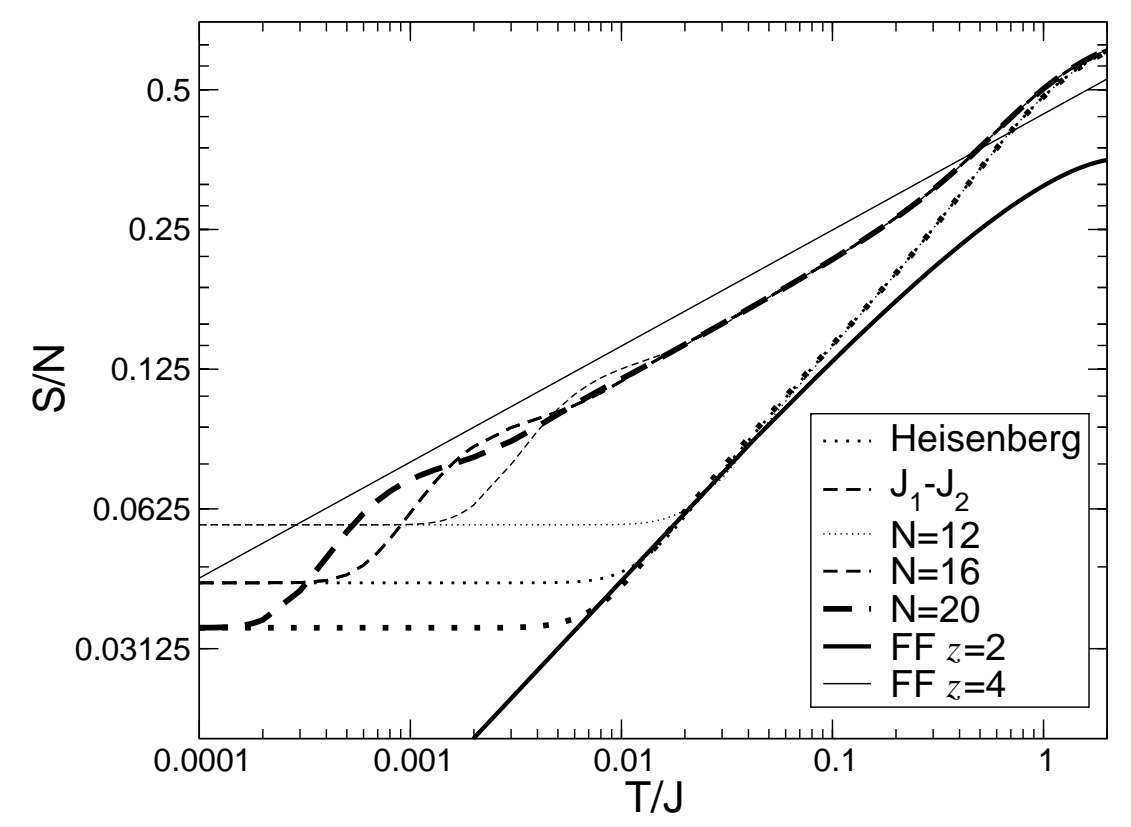

Figure 2. Numerical results for the entropy of the spin- $1 / 2$ Heisenberg chain (dotted lines) and the $J_{1}-J_{2}$ chain with $J_{1}=J, J_{2}=\frac{1}{4} J$ (dashed lines) at the saturation field $H_{c}=2 J$. The behaviour for a free fermion gas $(\mathrm{FF})$ is shown by the bold solid line, eq. (21), and the thin solid line, eq. (20) with $z=4$ and $A=\frac{1}{8} J$.

This fits the numerical data nicely in a wide temperature range $0.007 \lesssim T / J \lesssim 0.2$. Deviations from (21) for lower temperatures are determined by finite-size effects in the numerical data, whereas at higher temperatures further corrections to (21) become important. Although indeed the agreement at higher temperatures can be improved by including the next correction $O\left(T^{3 / 2}\right)$, we will not pursue this further here.

The thin sold line in Fig. 2 shows eq. (20) with $z=4$ and $A=\frac{1}{8} J$ appropriate for the frustrated chain. Comparison with the numerical data for intermediate temperatures confirms the expected power-law behaviour $S \sim T^{1 / 4}$. However, the prefactor derived from eq. (20) for $z=4$ is by about $20 \%$ too big in contrast to the Heisenberg chain $(z=2)$. This behaviour of the prefactor reflects the fact that interactions are relevant for $z=4$ and partially reduce the entropy compared to the free-gas expression eq. (20).

\section{Anisotropic $X Y$ chain in transverse field}

Low crystal symmetry of real (quasi) one-dimensional magnetic materials is compatible with various types of spin anisotropy. If the magnetic field is applied in a general direction, the anisotropy leads to a nontrivial behaviour by breaking the conservation of the total magnetization. It is, therefore, important to investigate the effect of spin anisotropy on the magnetocaloric effect. The simplest kind of anisotropy is anisotropic exchange interaction. We consider the following Hamiltonian

$$
\hat{\mathcal{H}}=\sum_{i}\left(J_{x} S_{i}^{x} S_{i+1}^{x}+J_{y} S_{i}^{y} S_{i+1}^{y}-H S_{i}^{z}\right),
$$




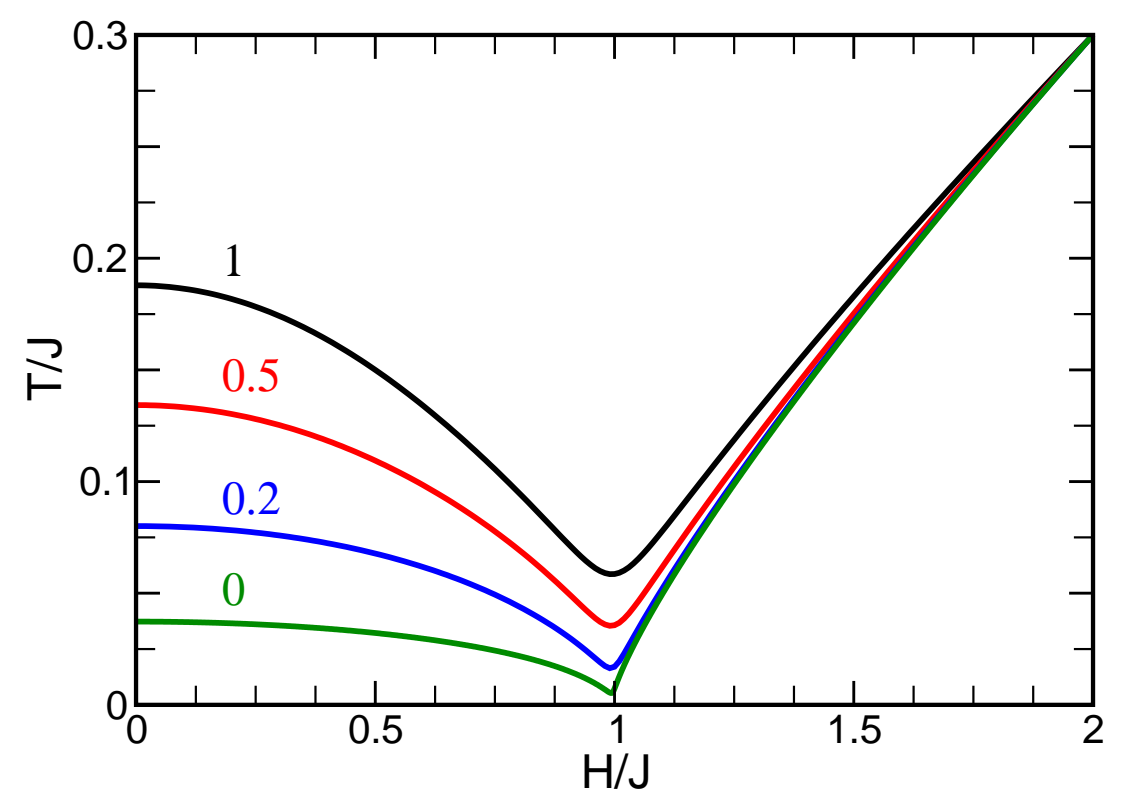

Figure 3. Dependence of the magnetocaloric effect on anisotropy. The number near each curve indicates the corresponding value of the anisotropy parameter $\gamma$.

which at $J_{x}=J_{y}$ reduces to the $X Y$ chain and at $J_{y}=0$ describes an Ising chain in a transverse field. Introducing new parameters $J=\frac{1}{2}\left(J_{x}+J_{y}\right)$ and $\gamma=\left(J_{x}-J_{y}\right) /\left(J_{x}+J_{y}\right)$ and applying the Jordan-Wigner transformation, the Hamiltonian (22) is reduced to

$$
\hat{\mathcal{H}}=-\frac{H N}{2}+\sum_{k}(H+J \cos k) c_{k}^{\dagger} c_{k}+\frac{i \gamma J}{2} \sin k\left(c_{k}^{\dagger} c_{-k}^{\dagger}-c_{-k} c_{k}\right) .
$$

The Bogoliubov transformation from original fermion operators $c_{k}$ to new operators $a_{k}$ :

$$
c_{k}=u_{k} a_{k}+i v_{k} a_{-k}^{\dagger}, \quad c_{-k}=u_{k} a_{-k}-i v_{k} a_{k}^{\dagger}
$$

diagonalizes the quadratic Hamiltonian (23) under the two conditions: (i) $u_{k}^{2}+v_{k}^{2}=1$ and (ii)

$$
2 u_{k} v_{k}=-\frac{\gamma J \sin k}{\sqrt{(H+J \cos k)^{2}+\gamma^{2} J^{2} \sin ^{2} k}} .
$$

The transformed Hamiltonian has a simple diagonal form:

$$
\hat{\mathcal{H}}=\sum_{k} \varepsilon_{k}\left(a_{k}^{\dagger} a_{k}-\frac{1}{2}\right), \quad \varepsilon_{k}=\sqrt{(H+J \cos k)^{2}+\gamma^{2} J^{2} \sin ^{2} k} .
$$

The anisotropic $X Y$ chain (22) has a phase transition at $H_{c}=J$. Near the transition point the long-wavelength spectrum is given by $\left(\Delta_{H}=H-H_{c}\right)$

$$
\varepsilon_{\pi+q} \approx \sqrt{\Delta_{H}^{2}+\gamma^{2} J^{2} q^{2}}
$$

The dispersion acquires a relativistic form at $H=H_{c}: \varepsilon_{\pi+q} \approx \gamma J q$. The expressions for the free energy, entropy and the specific heat are the same as for a gas of free fermions. The magnetization of the anisotropic $X Y$ chain is

$$
M=\sum_{k} \frac{H+J \cos k}{\varepsilon_{k}}\left(\frac{1}{2}-n_{k}\right) \text {. }
$$


At zero temperature $n_{k} \equiv 0$ and the total magnetization does not saturate above $H_{c}$. In the case of the Ising chain $\gamma=1$, the magnetization has a universal value $M\left(H_{c}\right)=1 / \pi$ at $T=0$. The square-root singularity near $H_{c}$ is replaced with a weaker logarithmic behaviour: $\partial M / \partial H \sim \ln \left(\pi \gamma / \Delta_{H}\right)$.

The expression for the magnetocaloric effect changes compared to the $X Y$ chain

$$
\left(\frac{\partial S}{\partial H}\right)_{T}=-\frac{1}{T^{2}} \sum_{k}(H+J \cos k) n_{k}\left(1-n_{k}\right) .
$$

Due to a linear dispersion law the density of low-energy states in the anisotropic chain remains finite instead of the square-root divergence typical for isotropic spin chains. As a result, the entropy at $H=H_{c}$ depends linearly on temperature:

$$
S=\frac{\pi T}{6 \gamma J} .
$$

This leads to a reduction of the magnetocaloric effect compared to the isotropic case. The cooling rate at the saturation field also changes its temperature dependence for the anisotropic chain:

$$
\begin{array}{ll}
\left(\frac{\partial T}{\partial H}\right)_{S}^{H=H_{c}}=\frac{T}{2 \gamma^{2}} & \text { anisotropic } X Y, \\
\left(\frac{\partial T}{\partial H}\right)_{S}^{H=H_{c}}=\frac{2 c_{1}}{3 \zeta\left(\frac{3}{2}\right)\left(1-\frac{1}{\sqrt{2}}\right)} & \text { isotropic } X Y .
\end{array}
$$

The dependence of the adiabatic demagnetization process on the anisotropy is shown in Fig. 3] The lowest temperature reachable at $H \approx H_{c}$ grows significantly from the isotropic case $\gamma=0$ to the strongest anisotropy $\gamma=1$. Nevertheless, even an Ising chain exhibits sizeable variations of temperature. Another difference with the isotropic chain is a stronger temperature increase below $H_{c}$. This is related to a gap opening in the anisotropic chain, which leads to a significant reduction of entropy.

\section{Universal properties}

Universal low-temperature scaling at second-order quantum phase transitions is related to the dispersion of gapless excitations at the transition point. In particular, the scaling laws (28), (29) remain valid in the general case: $(\partial T / \partial H)_{S}^{H=H_{c}}=O(1)$ for

$U(1)$-symmetry about the field direction and $(\partial T / \partial H)_{S}^{H=H_{c}}=O(T)$ for $\mathbb{Z}_{2}$-symmetry about the field. In fact, this is not only true close to the saturation field, but also in the vicinity of plateaux in the magnetization curve [9,21,22. Since these field-induced transitions are universal (at least in one dimension), the scaling laws given by eqs. (28) and (2.9) should apply to generic second-order transitions in one dimension.

Our results can be related to a general scaling analysis of the magnetocaloric effect close to quantum critical points [23. First we note that e.g. the power laws in eqs. (15), (91), (20) and (27) can be written as $C, S \propto T^{d / z}$ at $H=H_{c}$ and $C$, 
$S \propto T^{y_{0}}\left|H-H_{c}\right|^{\nu\left(d-y_{0} z\right)}$ for $H \neq H_{c}$, using the notations of [23]. The case of $U(1)-$ symmetry is described by the exponents $(d=1)$

$$
z=2, \quad \nu=\frac{1}{2},
$$

$y_{0}=1$ in a gapless phase and $y_{0}=\infty$ in a massive phase. For the case of $\mathbb{Z}_{2}$-symmetry the exponents are given by $(d=1)$

$$
z=1, \quad \nu=1, \quad y_{0}=\infty .
$$

With these identifications, our results (10) and (12) for the isotropic $X Y$ chain are consistent with the predictions from general scaling arguments [23. Furthermore, eq. (29) obeys the general scaling form $(\partial T / \partial H)_{S}^{H=H_{c}} \propto T^{1-1 /(\nu z)}$.

The situation with only $\mathbb{Z}_{2}$-symmetry is an exception in so far as the general scaling arguments [23] do not directly lead to eq. (28). In this case, the leading term cancels at $H=H_{c}$ and eq. (28) is obtained from the next order in $T$. For $\mathbb{Z}_{2}$-symmetry, $H \neq H_{c}$ and $T \ll\left|H-H_{c}\right|$, the general scaling predictions [23] amount to

$$
\frac{1}{T}\left(\frac{\partial T}{\partial H}\right)_{S}=\frac{1}{H-H_{c}},
$$

which coincides with the result for the $U(1)$-symmetric system in the gapped state eq. (10) and agrees with an explicit computation for the anisotropic $X Y$ chain.

\section{Sawtooth chain}

The class of models with localized magnon excitations at the saturation field includes the spin-1/2 Heisenberg models on kagome and pyrochlore lattices [7,8,9]. In this section we study the magnetothermodynamics of a one-dimensional representative of this class, namely the sawtooth chain (called also a $\Delta$ chain). The sawtooth chain is a chain of corner-sharing triangles with two different exchanges for the base-base and base-vertex edges, see Fig. 目. For the special ratio of the coupling constants $J_{2}=\frac{1}{2} J_{1}$ the sawtooth chain supports localized magnon excitations, giving rise to a plateau in the $T=0$ magnetization curve at half the saturation field followed by a jump to saturation in the spin- $1 / 2$ model [7] 8. Below we always assume the choice of parameters $J_{2}=\frac{1}{2} J_{1}$ with $J_{1} \equiv J$.

In the fully saturated phase at high magnetic fields the sawtooth chain has two branches of single spin-flip excitations with energies

$$
\varepsilon_{1 k}=H-2 J, \quad \varepsilon_{2 k}=H-\frac{J}{2}(1-\cos k) .
$$

Excitations from the low-energy dispersionless branch correspond in real space to

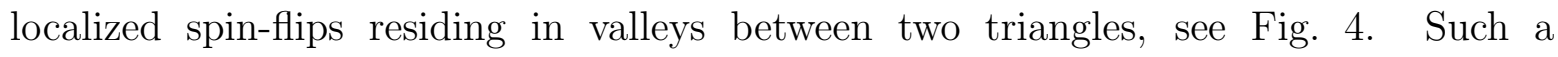
localization is a direct consequence of the frustration imposed by triangular topology and resembles to a large extent similar behaviour of magnons in kagome, pyrochlore and other frustrated lattices. Localized magnons in the sawtooth chain condense below the saturation field $H_{c}=2 J$ and form at zero temperature a dense magnon crystal 


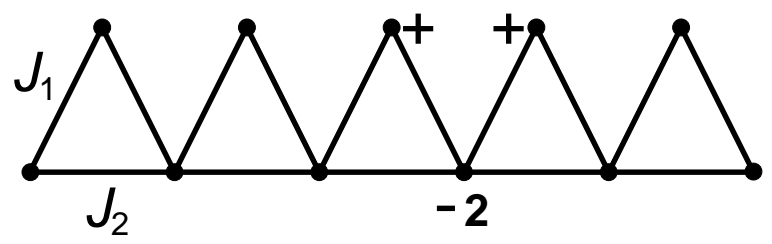

Figure 4. The sawtooth chain. Localized magnons, which exist for $J_{1}=2 J_{2}$, are trapped within one valley between two adjacent triangles with amplitudes indicated near each site.

with one spin-flip occupying every second valley between triangles [7, 8]. The magnon crystal ground state is two-fold degenerate and breaks the translational symmetry. In the following we discuss the low-temperature thermodynamics of the sawtooth chain in the vicinity of the saturation field, which is dominated by excitations from the lower branch.

We consider a chain which consists of $L$ triangles ( $N=2 L$ spins). The lowest energy of a state with $n$ excited magnons, $n \leq L / 2$, is equal to $n \varepsilon, \varepsilon=H-H_{c} \equiv \varepsilon_{1 k}$. The corresponding states are formed by nonoverlapping localized spin-flips, which occupy spatially separated valleys. This suggests a hard-dimer representation of the lowest energy $n$-magnon states so that the presence of a dimer on a given lattice site of the

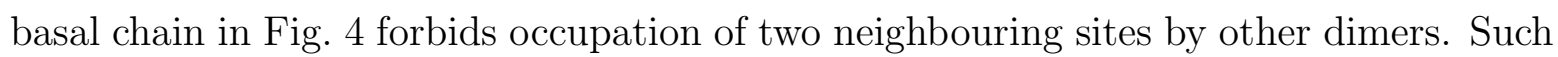
a simple construction of the lowest energy states in every $n$-magnon sector is complicated by the fact that the corresponding states are not orthogonal due to an overlap of the wave-functions of spin-flips trapped in two adjacent valleys. The states are, however, linearly independent and can be properly orthogonalized. Besides such lowest-energy localized states there are also scattering states in every $n$-magnon sector. The scattering states of $n$ magnons are separated from $n \varepsilon$ by a finite gap $\Delta_{n}$. For the two-magnon sector of the sawtooth chain the variational calculations estimate $\Delta_{2} \approx 0.44 \mathrm{~J}$ and suggest $\Delta_{n}=O\left(\Delta_{2}\right)$ in other sectors [24]. The present numerical calculations confirm this picture yielding $\Delta_{2} \simeq 0.42 \mathrm{~J}$, whereas for the magnon crystal state on the $1 / 2$-plateau $\Delta_{L / 2} \simeq 0.25 \mathrm{~J}$. Neglecting at low temperatures exponentially small corrections from the scattering states, we are left with the calculation of the free energy of hard dimers. This is a classical problem without dynamics and can be calculated exactly (see [24,25]). The classical partition function of the hard dimers is

$$
Z=\sum_{\{\sigma\}} \exp \left[-\frac{\varepsilon}{T} \sum_{i} \sigma_{i}\right] \prod_{\langle i j\rangle}\left(1-\sigma_{i} \sigma_{j}\right),
$$

where the index $i$ runs over the sites of the basal chain and $\sigma_{i}=0,1$ denotes the absence or presence of a dimer on a given site. The exclusion principle for nearest-neighbour sites is imposed by the last term. The partition function (34) can be rewritten as $Z=\operatorname{tr} \mathcal{T}^{L}$, 
$\mathcal{T}$ being the individual transfer matrix given by

$$
\mathcal{T}\left(\sigma, \sigma^{\prime}\right)=\left(\begin{array}{cc}
1 & \mathrm{e}^{-\varepsilon / 2 T} \\
\mathrm{e}^{-\varepsilon / 2 T} & 0
\end{array}\right) .
$$

This immediately yields the free energy and the entropy normalized per triangle

$$
\begin{aligned}
& F / L=-T \ln \left(\frac{1}{2}+\sqrt{\frac{1}{4}+\mathrm{e}^{-\varepsilon / T}}\right), \\
& S / L=\ln \left(\frac{1}{2}+\sqrt{\frac{1}{4}+\mathrm{e}^{-\varepsilon / T}}\right)+\frac{\varepsilon}{4 T}\left(2-\frac{1}{\sqrt{\frac{1}{4}+\mathrm{e}^{-\varepsilon / T}}}\right) .
\end{aligned}
$$

At $H=H_{c}$ the total entropy is

$$
S / L=\ln \left(\frac{1+\sqrt{5}}{2}\right) \approx 0.481212,
$$

which amounts to $34.7 \%$ of the total entropy $2 \ln 2$ of the spin- $1 / 2$ sawtooth chain. The same zero-temperature entropy of the sawtooth chain at $H=H_{c}$ has been independently obtained in [10. It is also known to express the entropy of an Ising antiferromagnetic chain at the saturation field [26]. The entropy $S_{H=H_{c}}$ has no temperature dependence in the hard-dimer approximation. A temperature dependence does arise from neglected multimagnon states and its magnitude can test the validity of the hard-dimer representation for the low temperature thermodynamics of the sawtooth chain.

The results on the temperature dependence of the entropy obtained by exact diagonalization for $N=20$ sites and by the hard-dimer mapping are compared in Fig. 5 for several magnetic fields. For $H \geq H_{c}=2$, the numerical results are in excellent agreement with the hard-dimer approximation at low temperatures consistent with a gap $\Delta \sim 0.2 J$ to states neglected in the hard-dimer picture. The magnon crystal arising for $H<H_{c}$ is two-fold degenerate. Hence, one obtains a non-extensive entropy $S=\ln 2$ at $T=0$, giving rise to finite-size effects in $S / N$ for $H<H_{c}$ (see numerical curve for $H=1.9$ in Fig. (5). In this regime, the hard-dimer approach is superior at low temperatures since it does not suffer from finite-size effects. The inset of Fig. 5 shows the field dependence of the specific heat at $T=0.05 \mathrm{~J}$. For $H \geq H_{c}$, the hard-dimer approximation agrees very well with the numerical results for $N=20$. For $H<H_{c}$, both approaches become approximate since the numerical result (full line) is affected by finite-size effects and the hard-dimer approximation neglects states that are relevant in this regime. The two peaks structure in the specific heat on both sides of the critical field obtained in the two approaches is typical for field-transitions with finite entropy at $H=H_{c}[24$.

\section{Comparison of different degrees of frustration}

In the preceding sections we have considered the spin- $1 / 2$ Heisenberg model, the $J_{1}-J_{2}$ chain at $J_{2}=\frac{1}{4} J_{1}$, and the sawtooth chain at $J_{2}=\frac{1}{2} J_{1}$. These models can be considered 


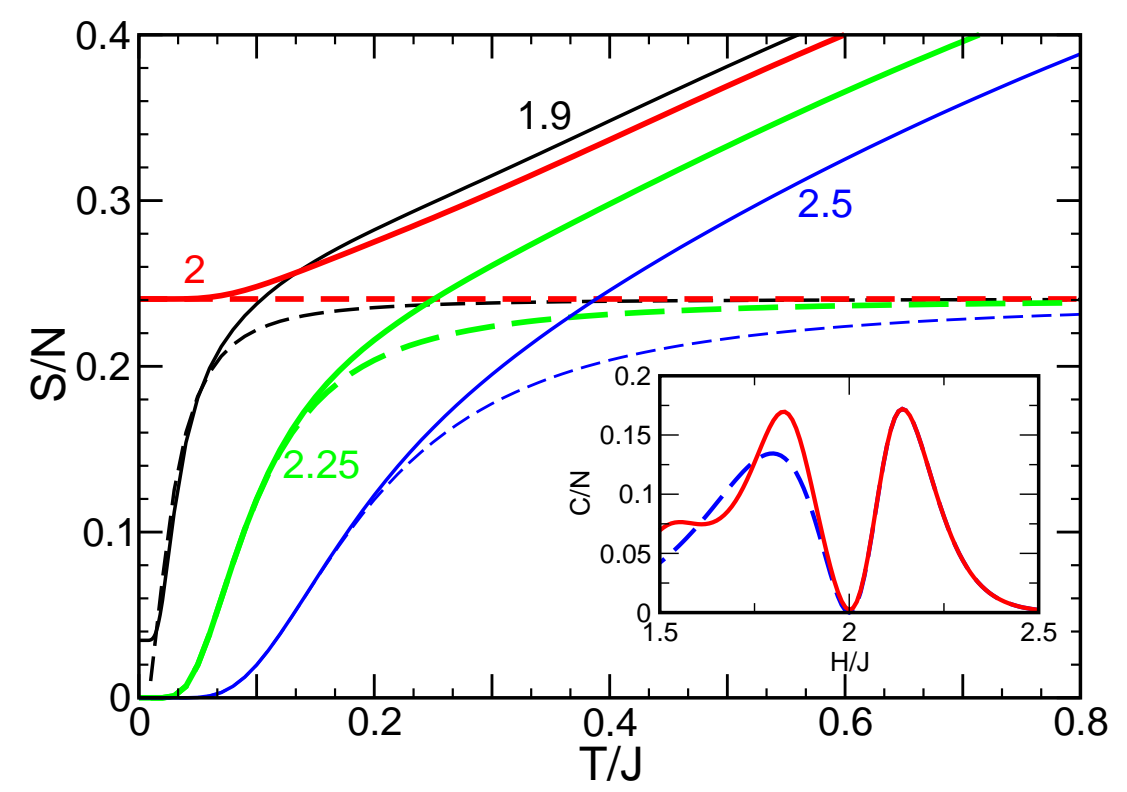

Figure 5. The temperature dependence of entropy for the sawtooth chain for different magnetic fields. Full lines are numerical results for $N=20$ sites, dashed lines are from the hard-dimer approximation. The number near each line indicates the corresponding value of the external field. The inset shows the field dependence of the specific heat $C$ at $T=0.05 \mathrm{~J}$ from numerical (full line) and the hard-dimer (dashed line) calculations.

as examples for increasing degree of frustration, as is reflected e.g. by an increase of the entropy at low temperatures at the saturation field $H_{c}$. In this section we will compare their entropy and adiabatic (de)magnetization curves in more detail.

Fig. [6 shows the entropy as a function of temperature and magnetic field for the Heisenberg chain, the $J_{1}-J_{2}$ chain with $J_{1}=J, J_{2}=\frac{1}{4} J$, and the sawtooth chain with $J_{1}=J, J_{2}=\frac{1}{2} J$. The saturation field is $H_{c}=2 J$ in all three cases. The contour lines show curves of constant entropy, i.e. adiabatic (de)magnetization curves. The global behaviour of all three models exhibits some similarities, in particular at high temperatures or sufficiently high above the saturation field. The main difference occurs at low temperatures and close to the saturation field, where the entropy clearly increases from the Heisenberg chain (a) to the $J_{1}-J_{2}$ chain (b) and further to the sawtooth chain (c) - compare also Figs. 2 and 5 for more detailed data exactly at $H_{c}$. The increase of entropy enhances the magnetocaloric effect as the magnetic field is swept through saturation.

The data in Fig. [6 has been computed numerically for $N=20$ sites. Hence, it is important to discuss finite-size effects. Some related comments for the entropy at the saturation field have already been made in the context of Figs. 2 and 5 . We now examine finite-size effects for all fields, focusing on the example of the sawtooth chain. Fig. [7]corresponds to a part of Fig. 66 (c) at low temperatures and fields and shows curves of constant entropy for $N=12,16$ and 20. The lowest curves start at $S / N=0.05$ and increase in steps of 0.05 . At low temperatures and close to $H_{c}=2 J$ we can compare with 

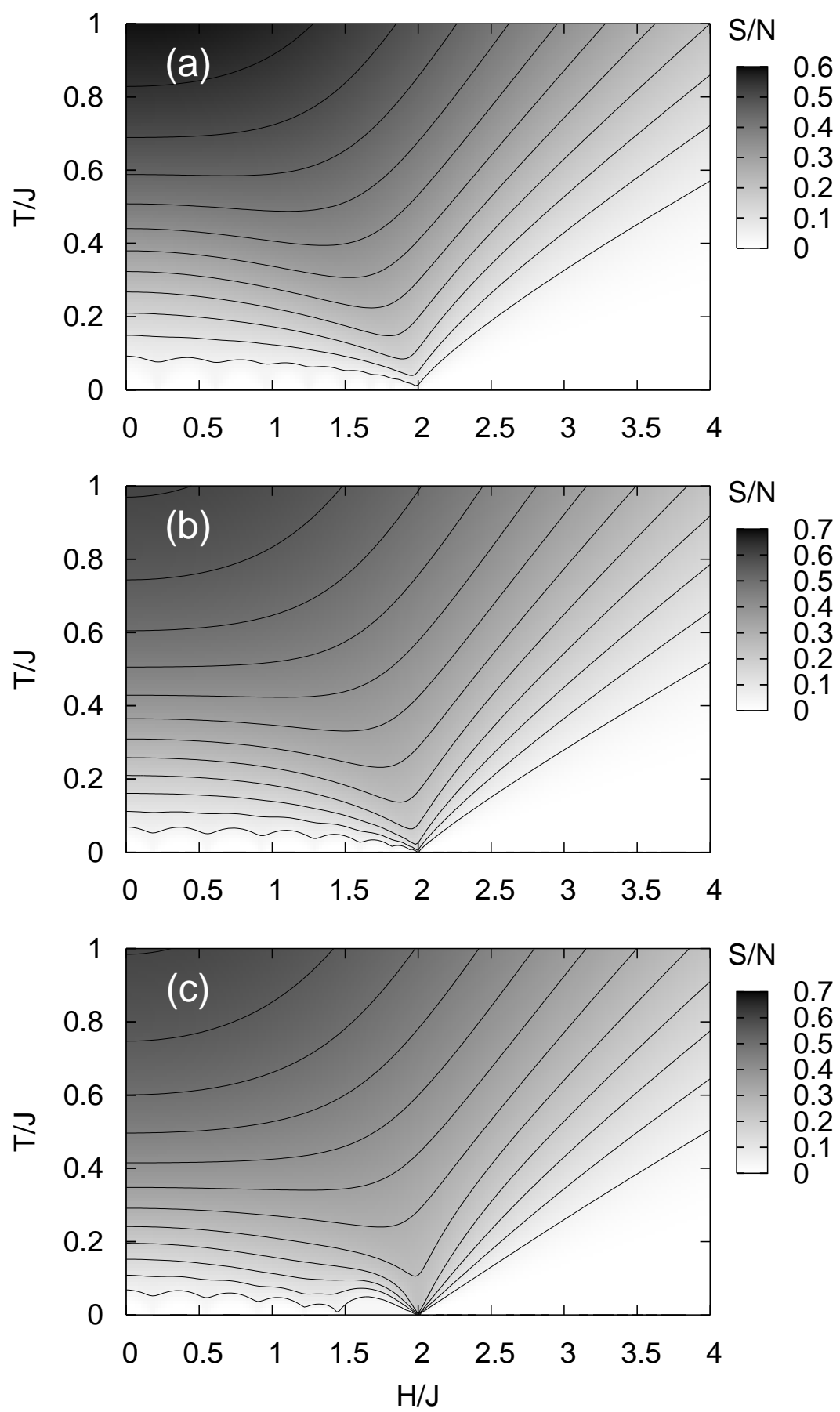

Figure 6. Entropy of $N=20$-sites lattices as a function of temperature and magnetic field for spin-1/2 models: (a) the Heisenberg chain, (b) the $J_{1}-J_{2}$ chain with $J_{1}=J$, $J_{2}=\frac{1}{4} J$, and (c) the sawtooth chain with $J_{1}=J, J_{2}=\frac{1}{2} J$. Lines show curves of constant entropy starting at the bottom with $S / N=0.05$ and increasing in steps of 0.05 . 


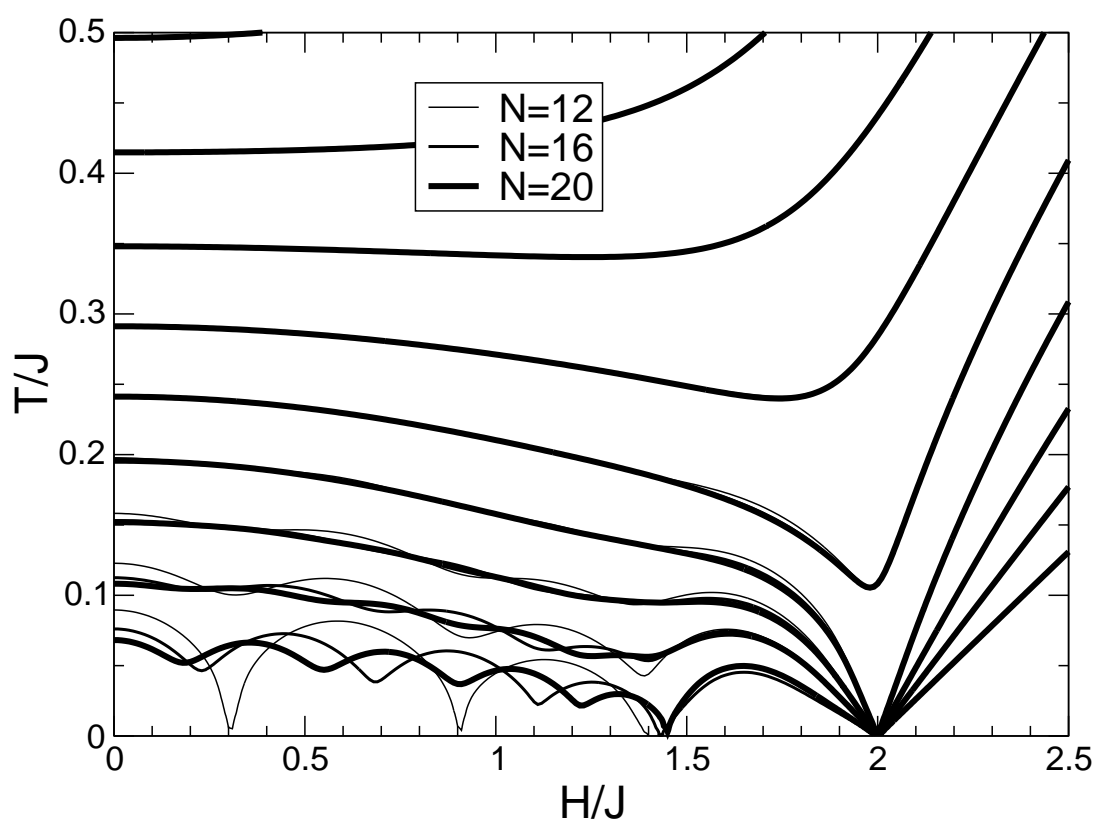

Figure 7. Lines of constant entropy, i.e. adiabatic (de)magnetization curves for the sawtooth chain with $J_{1}=J, J_{2}=\frac{1}{2} J$ for $N=12,16$ and 20 .

eq. (36). The corresponding lines of constant entropy are straight lines since the entropy of hard dimers depends on $H$ and $T$ only via the combination $\left(H-H_{c}\right) / T$. The lines obtained from eq. (36) lie on top of the lines obtained numerically for $N=20$ in Fig. 7 in the regime where the latter are linear. Since eq. (36) is valid for the thermodynamic limit, this means that finite-size effects in Fig. 7 are within the width of the lines for $N=20$ for $H$ close to $H_{c}$. Further comparison of the curves for different system sizes shows that the adiabatic demagnetization curves of a sawtooth chain with $N=20$ sites are essentially free of finite-size effects, except for $H \lesssim 1.7 \mathrm{~J}, T \lesssim 0.15 \mathrm{~J}$. These finitesize effects are related to the recovery of a gapless spectrum at $T=0$ and for $H \lesssim 1.5 \mathrm{~J}$. Similarly, finite-size effects can also be neglected for the Heisenberg chain and the $J_{1}-J_{2}$ chain (Fig. 6 (a) and (b)) except for $H<2 J, T \lesssim 0.15 J$. Note that in the gapless regimes $\left(H<H_{c}\right)$ temperature varies only weakly with the applied field (compare also Fig. (1).

Finally, Fig. 8 presents a direct comparison of adiabatic demagnetization curves for the spin-1/2 Heisenberg chain, the $J_{1}-J_{2}$ chain and the sawtooth chain. We start with all systems at the same temperature and a magnetic field noticeably above the saturation field $(H=3)$. During adiabatic demagnetization, all three systems cool upon approaching the saturation field. Clearly, the $J_{1}-J_{2}$ chain cools down to lower temperatures than the spin-1/2 Heisenberg chain under the same conditions, and the sawtooth chain to even lower temperatures. Along with numerical results we show by thin lines the analytic results for a free-fermion model for the Heisenberg chain and the $J_{1}-J_{2}$ chain. One observes that free fermions yield an excellent description of the Heisenberg chain above the saturation field while slightly bigger deviations for the $J_{1}-J_{2}$ 


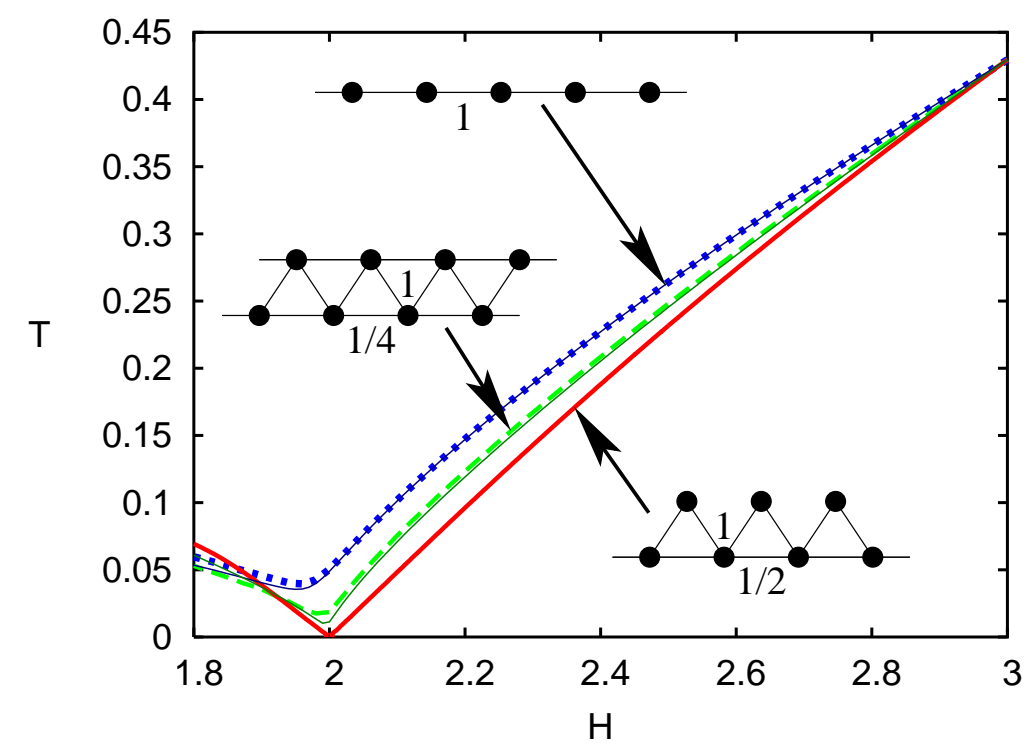

Figure 8. Comparison of adiabatic demagnetization curves when approaching the saturation field from above for the spin-1/2 Heisenberg chain, the $J_{1}-J_{2}$ chain with $J_{1}=1, J_{2}=1 / 4$, and for the sawtooth chain with $J_{1}=1, J_{2}=1 / 2$, as indicated in the figure. The three bold curves have been computed numerically for $N=20$ sites, the two thin curves have been obtained in a free-fermion approximation.

chain are due to the relevance of interactions in the latter case.

The adiabatic demagnetization curve in Fig. 8 of the sawtooth chain corresponds to an entropy below the value given in eq. (37). Hence, one even observes cooling to $T \rightarrow 0$ as $H \rightarrow H_{c}=2$. Nevertheless, the example of Fig. 8 is still at comparably high temperatures (entropy). Among others, this ensures that finite-size effects can be neglected although the bold curves in Fig. 8 have been computed with $N=20$. While asymptotic low-temperature expressions like eq. (11) are not accurate in this regime, the enhancement of the magnetocaloric effect with increasing frustration is evident.

\section{Conclusions and outlook}

When discussing the magnetocaloric effect at low temperatures, one should clearly distinguish two different cases: (i) large relative variations of entropy in a magnetic field, while the total entropy tends to zero, and (ii) large absolute variations of entropy. Let us first discuss the former case, which appears in ordinary nondegenerate quantum spin chains. We have shown that an enhanced magnetocaloric effect exists in the vicinity of quantum phase transitions in a magnetic field. Specifically, we have studied the transition at the saturation field. There are, however, many other systems/transitions to which our predictions apply, in particular second-order transitions at the boundaries of magnetization plateaux [9,21]. Field-induced transitions in 1D systems with a singlet ground state, like spin ladders or Haldane gap chains, are one specific example (see 
e.g. 21 for a recent review). Triplet excitations with $\Delta S^{z}=+1$ condense, when the Zeeman energy exceeds the triplet gap. Triplets can be represented as hard-core bosons, which are mapped in one dimension to weakly interacting spinless fermions [12, 16]. Therefore, the entropy of triplet excitations close to the transition field is also described by the equations (92) in the single-particle approximation. A less trivial example is the Bose condensation of magnetic excitations in 2D spin systems with 1D type degeneracies in the energy spectra. Such a possibility occurs in the $J_{1}-J_{2}$ antiferromagnetic model on a square lattice near the saturation field [27] as well as in the 2D singlet gap magnet $\mathrm{Cs}_{3} \mathrm{Cr}_{2} \mathrm{Br}_{9}$ [28]. Triplet excitations above the singlet gap have also a large degeneracy in the singlet state of $\mathrm{SrCu}_{2}\left(\mathrm{BO}_{3}\right)_{2}$ [29, 30] which should lead to a large entropy release at the gap closing transition. In the frustrated antiferromagnetic chain the magnetocaloric effect is enhanced due to a cancellation of the leading $q^{2}$ term in the one-particle dispersion (19) for $J_{2} / J_{1}=4$. Such a cancellation remains valid for ferromagnetic sign of the nearest-neighbour exchange $J_{1}<0$. The magnetization curve of the $J_{1}-J_{2}$ chain with ferromagnetic $J_{1}<0$ is also particularly steep below the saturation (see e.g. Fig. 4 of [20]), suggesting that the magnetocaloric effect may also be enhanced in this regime. $\mathrm{Rb}_{2} \mathrm{Cu}_{2} \mathrm{Mo}_{3} \mathrm{O}_{12}$ is a candidate for a realization in the latter parameter regime with a moderate saturation field [31].

A preliminary experimental indication of a large magnetocaloric effect has been obtained in pulsed field measurements in a spin- $1 / 2$ Heisenberg chain antiferromagnet [32. In the vicinity of the saturation field the magnetization curve deviates significantly from the behaviour expected for the corresponding bath temperature indicating a sizeable magnetocaloric effect. Thus, the magnetocaloric effect can significantly affect experimental results obtained in pulsed magnetic fields. A systematic experimental investigation of the magnetocaloric effect near quantum phase transitions can also be used with the help of eqs. (28), (29) to clarify the role of spin anisotropies in a given magnetic material.

Large absolute variations of entropy in a magnetic field appear for a class of geometrically frustrated antiferromagnetic models. The sawtooth chain considered in sections 8 and 9 has a finite zero-temperature entropy at the saturation field. The analysis of the magnetothermodynamics of this model shows that in a strongly correlated spin system there are emergent "paramagnetic" degrees of freedom, which experience an effective magnetic field $h=\left(H-H_{c}\right)$. Such effective "paramagnetic moments" are responsible for an enhanced cooling power in the vicinity of $H_{c}$ very similar to standard paramagnetic salts [2]. However, the "paramagnetic" language is not completely adequate and a picture of hard localized magnons is more appropriate for the physical description. Among the differences with a simple paramagnetic picture, we mention the geometrical origin of the finite entropy: the zero- $T$ entropy at the saturation field does not depend on the value of the local spin. Also, there is an asymmetry between the $h>0$ and $h<0$ regions close to the saturation field in Fig. 7 .

The antiferromagnetic sawtooth chain model is realized in delafossite $\mathrm{YCuO}_{2.5}$ [33, though with a yet unknown ratio of the exchange constants $J_{2} / J_{1}$. Our theoretical 
study should also be relevant for 2D and 3D spin models on kagome, pyrochlore and garnet (hyper-kagome) lattices, which have numerous experimental realizations. The magnetocaloric effect has been known for a long time in gadolinium gallium garnet $\mathrm{Gd}_{3} \mathrm{Ga}_{5} \mathrm{O}_{12}$, which is often utilized in adiabatic demagnetization refrigerators 34, 35. Very recently, a large magnetocaloric effect has also been measured in the pyrochlore antiferromagnet $\mathrm{Gd}_{2} \mathrm{Ti}_{2} \mathrm{O}_{7}$ [36. Magnetic $\mathrm{Gd}^{3+}$ ions have a rather large spin $S=7 / 2$. This justifies the classical approximation applied for pyrochlore and garnet antiferromagnets in [6]. Still, the classical model cannot describe the behaviour at low temperatures $T \lesssim J S$. One of the physical implications of the present work is that the entropy on frustrated lattices has a purely geometrical origin. Therefore, $S=1 / 2$ frustrated magnetic materials should be as good as $S \gg 1 / 2$ compounds for cooling by adiabatic demagnetization, contrary to the experience with paramagnetic salts [2]. We suggest that a synthesis of new spin- $1 / 2$ antiferromagnetic materials with kagome, pyrochlore and garnet lattices should be interesting both from a fundamental point of view and with regard to possible technological applications in the field of lowtemperature magnetic cooling.

\section{Acknowledgments}

We would like to thank G. Jackeli, A. Klümper, J. Richter, A. Rosch, H. Tsunetsugu, and S. Wessel for useful discussions. The more complicated numerical computations presented in this article have been performed on the compute-server cfgauss at the computing center of the TU Braunschweig. A.H. would like to acknowledge the hospitality of the Institute for Theoretical Physics of the University of Hannover during the course of this work.

\section{References}

[1] M. Cyrot, Magnetism of metals and alloys (North Holland, Amsterdam, 1982).

[2] A. M. Tishin, Magnetocaloric effect in the vicinity of phase transitions, in Handbook of Magnetic Materials v. 12, edited by K.H.J. Buschow (Elsevier, Amsterdam, 1999).

[3] O. V. Lounasmaa, Experimental Principles and Methods Below $1 \mathrm{~K}$ (Academic Press, London, 1974).

[4] V. K. Pecharsky and K. A. Gschneidner, Phys. Rev. Lett. 78, 4494 (1997).

[5] O. Tegus, E. Brück, K. H. J. Buschow, and F. R. de Boer, Nature 415, 150 (2002).

[6] M. E. Zhitomirsky, Phys. Rev. B 67, 104421 (2003).

[7] J. Schulenburg, A. Honecker, J. Schnack, J. Richter, and H.-J. Schmidt, Phys. Rev. Lett. 88, 167207 (2002).

[8] J. Richter, J. Schulenburg, A. Honecker, J. Schnack, and H.-J. Schmidt, J. Phys.: Condensed Matter 16, S779 (2004).

[9] J. Richter, J. Schulenburg, and A. Honecker, Lect. Notes Phys. 645, 85-153 (2004)

[10] O. Derzhko and J. Richter, e-print: cond-mat/0404204

[11] M. E. Zhitomirsky, A. Honecker, and O. A. Petrenko, Phys. Rev. Lett. 85, 3269 (2000); M. E. Zhitomirsky, Phys. Rev. Lett. 88, 057204 (2002).

[12] M. Takahashi, Thermodynamics of one-dimensional solvable models (Cambridge University Press, Cambridge, 1999). 
[13] S. Katsura, Phys. Rev. 127, 1508 (1962).

[14] G. I. Dzhaparidze and A. A. Nersesyan, JETP Lett. 27, 334 (1978).

[15] V. L. Pokrovsky and A. L. Talapov, Phys. Rev. Lett. 42, 65 (1979).

[16] S. Sachdev, T. Senthil, and R. Shankar, Phys. Rev. B 50, 258 (1994).

[17] M. Takahashi, Prog. Theor. Phys. 50, 1519 (1973).

[18] M. Schmidt, C. Gerhardt, K.-H. Mütter, and M. Karbach, J. Phys.: Condensed Matter 8, 553 (1996).

[19] C. Gerhardt, K.-H. Mütter, and H. Kröger, Phys. Rev. B 57, 11504 (1998).

[20] D. C. Cabra, A. Honecker, and P. Pujol, Eur. Phys. J. B 13, 55 (2000).

[21] H.-J. Mikeska and A. K. Kolezhuk, Lect. Notes Phys. 645, 1-83 (2004)

[22] A. Honecker, The magnetization process of antiferromagnetic quantum spin systems and macroscopic quantum effects, habilitation thesis (2003).

[23] L. Zhu, M, Garst, A. Rosch, and Q. Si, Phys. Rev. Lett. 91, 066404 (2003).

[24] M. E. Zhitomirsky and H. Tsunetsugu, e-print: cond-mat/0405578

[25] R. J. Baxter, Exactly Solved Models in Statistical Mechanics (Academic Press, London, 1982).

[26] B. D. Matcalf and C. P. Yang, Phys. Rev. B 18, 2304 (1978).

[27] G. Jackeli and M. E. Zhitomirsky, Phys. Rev. Lett. 93, 017201 (2004).

[28] B. Leuenberger, H. U. Güdel, R. Feile, and J. K. Kjems, Phys. Rev. B 31, 597 (1985).

[29] H. Kageyama, K. Yoshimura, R. Stern, N. V. Mushnikov, K. Onizuka, M. Kato, K. Kosuge, C. P. Slichter, T. Goto, and Y. Ueda, Phys. Rev. Lett. 82, 3168 (1999).

[30] K. Totsuka, S. Miyahara, and K. Ueda, Phys. Rev. Lett. 86, 520 (2001).

[31] M. Hase, H. Kuroe, K. Ozawa, O. Suzuki, H. Kitazawa, G. Kido, and T. Sekine, e-print: cond-mat/0404463

[32] B. Wolf, S. Zherlitsyn, B. Lüthi, N. Harrison, U. Löw, V. Pashchenko, M. Lang, G. Margraf, H.-W. Lerner, E. Dahlmann, F. Ritter, W. Assmus, and M. Wagner, Phys. Rev. B 69, 092403 (2004).

[33] R. J. Cava, H. W. Zandenbergen, A. P. Ramirez, H. Takagi, C. T. Chen, J. J. Krajewski, W. F. Peck, J. V. Waszczak, G. Meigs, R. S. Roth, and L. F. Schneemeyer, J. Solid State Chem. 104, 437 (1993); K. Isawa, Y. Yaegashi, M. Komatsu, M. Nagano, and S. Sudo, Phys. Rev. B 56, 3457 (1997).

[34] G. E. Brodale, E. W. Hornung, R. A. Fisher, and W. F. Giauque, J. Chem. Phys. 62, 4041 (1975).

[35] J. A. Barclay and W. A. Steyert, Cryogenics 22, 73 (1982).

[36] S. S. Sosin, L. A. Prozorova, A. I. Smirnov, A. I. Golov, I. B. Berkutov, O. A. Petrenko, G. Balakrishnan, and M. E. Zhitomirsky, e-print: cond-mat/0404529. 